\title{
Strongly Starlike Functions and Related Classes
}

\author{
Mamoru Nunokawa and Janusz Sokół
}

\begin{abstract}
We consider univalent functions, analytic in the unit disc $|z|<1$ in the complex plane $\mathbb{C}$ which map $|z|<1$ onto a domain with some nice property. The purpose of this paper is to find some new conditions for strong starlikeness and some related results.
\end{abstract}

\section{Introduction}

Let $\mathcal{H}$ denote the class of analytic functions in the unit disc $\mathbb{D}=\{z:|z|<1\}$ in the complex plane $\mathbb{C}$. For $a \in \mathbb{C}$ and $n \in \mathbb{N}$ we denote by

$$
\mathcal{H}[a, n]=\left\{f \in \mathcal{H}: f(z)=a+a_{n} z^{n}+\cdots, a_{n} \neq 0\right\}
$$

and

$$
\mathcal{A}_{n}=\left\{f \in \mathcal{H}: f(z)=z+a_{n+1} z^{n+1}+\cdots\right\},
$$

so $\mathcal{A}=\mathcal{A}_{1}$. Let $\mathcal{S}$ be the subclass of $\mathcal{A}$ whose members are univalent in $\mathbb{D}$.

A set $E \subset \mathbb{C}$ is said to be starlike with respect to the point $0 \in E$ if and only if the linear segment joining the origin to every other point $w \in E$ lies entirely in $E$, while a set $E$ is said to be convex if and only if it is starlike with respect to each of its points. Let $\mathcal{S}^{*}$ denote the class of all functions $f \in \mathcal{S}$ such that the set $f(\mathbb{D})$ is starlike with respect 0 . A natural extension of the notion starlike is to be starlike of order $\alpha$. The class $\mathcal{S}_{\alpha}^{*}$ of starlike functions of order $\alpha<1$ may be defined as

$$
\mathcal{S}_{\alpha}^{*}=\left\{f \in \mathcal{A}: \mathfrak{R e} \frac{z f^{\prime}(z)}{f(z)}>\alpha, z \in \mathbb{D}\right\} .
$$

The class $\mathcal{S}_{\alpha}^{*}$ and the class $\mathcal{K}_{\alpha}$ of convex functions of order $\alpha<1$

$$
\mathcal{K}_{\alpha}:=\left\{f \in \mathcal{A}: \mathfrak{R e}\left(1+\frac{z f^{\prime \prime}(z)}{f^{\prime}(z)}\right)>\alpha, z \in \mathbb{D}\right\}
$$

2010 Mathematics Subject Classification. 30C45, 30C80.

Key words and phrases. univalent functions; starlike; convex; strongly starlike .

Corresponding author: Janusz Sokół. 


$$
=\left\{f \in \mathcal{A}: z f^{\prime} \in \mathcal{S}_{\alpha}^{*}\right\}
$$

introduced Robertson in [14], see also [5]. If $\alpha \in[0 ; 1)$, then a function in either of these sets is univalent, if $\alpha<0$ it may fail to be univalent. In particular we denote $\mathcal{S}_{0}^{*}=\mathcal{S}^{*}, \mathcal{K}_{0}=\mathcal{K}$, the classes of starlike and convex functions, respectively. Furthermore, note that if $f \in \mathcal{K}_{\alpha}$ then $f \in \mathcal{S}_{\delta(\alpha)}^{*}$, see [18], where

$$
\delta(\alpha)= \begin{cases}\frac{1-2 \alpha}{2^{2-2 \alpha}-2} & \text { for } \quad \alpha \neq \frac{1}{2} \\ \frac{1}{2 \log 2} & \text { for } \quad \alpha=\frac{1}{2} .\end{cases}
$$

Let $\mathcal{S S}^{*}(\beta)$ denote the class of strongly starlike functions of order $\beta, 0<\beta<2$,

$$
\mathcal{S S}^{*}(\beta):=\left\{f \in \mathcal{A}:\left|\arg \frac{z f^{\prime}(z)}{f(z)}\right|<\frac{\beta \pi}{2}, z \in \mathbb{D}\right\},
$$

which was introduced in [15] and [1], see also [9]. Furthermore, $\mathcal{S K}(\beta)=\left\{f \in \mathcal{A}: z f^{\prime} \in \mathcal{S S}^{*}(\beta)\right\}$ denote the class of strongly convex functions of $\operatorname{order} \beta$. Analogously to (1.1), in the work [8] it was proved that if $\beta \in(0,1)$ and $f \in \mathcal{S K}(\alpha(\beta))$, then $f \in \mathcal{S S}(\beta)$, where

$$
\alpha(\beta)=\beta+\frac{2}{\pi} \tan ^{-1}\left(\frac{\beta n(\beta) \sin (\pi(1-\beta) / 2)}{m(\beta)+\beta n(\beta) \cos (\pi(1-\beta) / 2)}\right),
$$

and where

$$
m(\beta)=(1+\beta)^{(1+\beta) / 2} n(\beta)=(1-\beta)^{(\beta-1) / 2} .
$$

The class $\mathcal{G}(\alpha, \gamma), \gamma>0,0<\alpha \leq 1$ of $\gamma$-strongly starlike functions of order $\alpha$ consists of functions $f \in \mathcal{A}$ satisfying

$$
\left|\arg \left\{\left(\frac{z f^{\prime}(z)}{f(z)}\right)^{1-\gamma}\left(1+\frac{z f^{\prime \prime}(z)}{f^{\prime}(z)}\right)^{\gamma}\right\}\right|<\frac{\alpha \pi}{2}, \quad z \in \mathbb{D},
$$

and such that

$$
f(z) f^{\prime}(z)\left(1+\frac{z f^{\prime \prime}(z)}{f^{\prime}(z)}\right) \neq 0, z \in \mathbb{D} \backslash\{0\} .
$$

Note that Lewandowski, S. Miller and Złotkiewicz 1974 [6] have introduced the class of $\gamma$-starlike functions, denoted here by $\mathcal{G}(1, \gamma)$, which satisfy (1.5) and such that

$$
\mathfrak{R e}\left\{\left(\frac{z f^{\prime}(z)}{f(z)}\right)^{1-\gamma}\left(1+\frac{z f^{\prime \prime}(z)}{f^{\prime}(z)}\right)^{\gamma}\right\}>0, z \in \mathbb{D} \text {. }
$$

Lemma 1.1. (Nunokawa [8]) Let $q(z)$ be in $\mathcal{H}[1, m]$ with $q(z) \neq 0$. If there exists a point $z_{0}$, $\left|z_{0}\right|<1$, such that $|\arg \{q(z)\}|<\pi \gamma / 2$ for $|z|<\left|z_{0}\right|$ and $\left|\arg \left\{q\left(z_{0}\right)\right\}\right|=\pi \gamma / 2$ for some $\gamma \in(0,2)$, then we have

$$
\frac{z_{0} q^{\prime}\left(z_{0}\right)}{q\left(z_{0}\right)}=\frac{2 i k \arg \left\{q\left(z_{0}\right)\right\}}{\pi}
$$

for some $k \geq m\left(a+a^{-1}\right) / 2 \geq\left(a+a^{-1}\right) / 2$, where $\left\{q\left(z_{0}\right)\right\}^{1 / \gamma}= \pm i a$, and $a>0$. 
It should be remarked that for interesting related results associated with Lemma 2.1, the authors may refer to [7]. In this paper we consider some problems related to Lemma 2.1, which may be called as Nunokawa's lemma.

\section{Main result}

Lemma 2.1. Let $\beta$ be in $(0,1)$. Then we have $\forall \beta \in(0,1)$ :

$$
\arg \left\{e^{i \pi(1-\beta) / 2}\left[\left(\frac{1+\beta}{1-\beta}\right)^{(1-\beta) / 2}+\left(\frac{1+\beta}{1-\beta}\right)^{-(1+\beta) / 2}\right]-1\right\} \leq \pi-\frac{\pi \beta}{2} .
$$

Proof. Observe that

$$
\forall \beta \in(0,1): 1<\left(\frac{1+\beta}{1-\beta}\right)^{(1-\beta) / 2}+\left(\frac{1+\beta}{1-\beta}\right)^{-(1+\beta) / 2}<2 .
$$

Therefore, a geometrical observation shows that for all $\beta \in(0,1)$, we have

$$
\begin{aligned}
& \arg \left\{e^{i \pi(1-\beta) / 2}\left[\left(\frac{1+\beta}{1-\beta}\right)^{(1-\beta) / 2}+\left(\frac{1+\beta}{1-\beta}\right)^{-(1+\beta) / 2}\right]-1\right\} \\
\leq & \arg \left\{e^{i \pi(1-\beta) / 2}-1\right\} \\
= & \frac{\pi}{2}+\frac{(1-\beta) \pi}{4} \\
< & \pi-\frac{\pi \beta}{2} .
\end{aligned}
$$

Theorem 2.1. Let $\beta$ be in $(0,1), p(z) \in \mathcal{H}[1,1]$, and suppose that

$$
\left|\arg \left\{\frac{z p^{\prime}(z)}{p(z)}-p(z)\right\}\right|>\frac{\pi \alpha(\beta)}{2}, \quad z \in \mathbb{D},
$$

then we have

$$
|\arg \{p(z)\}|<\frac{\pi \beta}{2}, \quad z \in \mathbb{D}
$$

where

$$
\tan \frac{\pi \alpha(\beta)}{2}=\tan \frac{\pi \beta}{2}+\frac{\beta}{(1-\beta) \cos (\pi \beta / 2)}\left(\frac{1-\beta}{1+\beta}\right)^{(1+\beta) / 2} .
$$

Proof. If there exists a point $z_{0},\left|z_{0}\right|<1$, such that $|\arg \{p(z)\}|<\pi \beta / 2$ for $|z|<\left|z_{0}\right|$ and $\left|\arg \left\{p\left(z_{0}\right)\right\}\right|=\pi \beta / 2$ for some $0<\beta<1$, then by Lemma 1.1 we have

$$
\frac{z_{0} p^{\prime}\left(z_{0}\right)}{p\left(z_{0}\right)}=\frac{2 i k \arg \left\{p\left(z_{0}\right)\right\}}{\pi}
$$


for some

$$
k \geq\left(a+a^{-1}\right) / 2,
$$

where

$$
\left\{p\left(z_{0}\right)\right\}^{1 / \beta}= \pm i a
$$

and $a>0$. Then, for the case $p\left(z_{0}\right)=a^{\beta}(\cos (\pi \beta / 2)+i \sin (\pi \beta / 2))$, we have from (2.5)

$$
\frac{z_{0} p^{\prime}\left(z_{0}\right)}{p^{2}\left(z_{0}\right)}-1=e^{i \pi(1-\beta) / 2} \beta k \frac{1}{a^{\beta}}-1
$$

Note that

$$
\beta k \frac{1}{a^{\beta}} \geq \frac{\beta}{2}\left(a^{1-\beta}+a^{-1-\beta}\right) .
$$

Let us put

$$
g(a)=\frac{1}{2}\left(a^{1-\beta}+a^{-1-\beta}\right), \quad a>0 .
$$

Then we have

$$
g^{\prime}(a)=\frac{1}{2}\left((1-\beta) a^{-\beta}-(1+\beta) a^{-2-\beta}\right),
$$

and so, $g(a)$ takes its minimum value at

$$
a=\sqrt{(1+\beta) /(1-\beta)}
$$

Furthermore, for the function

$$
H(\beta)=\left(\frac{1+\beta}{1-\beta}\right)^{(1-\beta) / 2}+\left(\frac{1+\beta}{1-\beta}\right)^{-(1+\beta) / 2}, \beta \in(0,1),
$$

the $H^{\prime}(\beta)$ decreases from 0 , when $\beta \in(0,1)$. Therefore, $H(\beta)$ is a decreasing function, from 2 to 1 :

$$
\lim _{\beta \rightarrow 1^{-}}=\left(\frac{1+\beta}{1-\beta}\right)^{(1-\beta) / 2}+\left(\frac{1+\beta}{1-\beta}\right)^{-(1+\beta) / 2}=1 .
$$

Therefore, from (2.7) and from (2.8), we have

$$
\begin{aligned}
\arg \left\{\frac{z_{0} p^{\prime}\left(z_{0}\right)}{p^{2}\left(z_{0}\right)}-1\right\} & =\arg \left\{e^{i \pi(1-\beta) / 2} \beta k \frac{1}{a^{\beta}}-1\right\} \\
& \leq \arg \left\{e^{i \pi(1-\beta) / 2}\left(\left(\frac{1+\beta}{1-\beta}\right)^{(1-\beta) / 2}+\left(\frac{1+\beta}{1-\beta}\right)^{-(1+\beta) / 2}\right)-1\right\} \\
& \leq \pi-\frac{\pi \beta}{2}
\end{aligned}
$$

because of Lemma 2.1. We also have

$$
\frac{z_{0} p^{\prime}\left(z_{0}\right)}{p\left(z_{0}\right)}-p\left(z_{0}\right)=p\left(z_{0}\right)\left\{\frac{z_{0} p^{\prime}\left(z_{0}\right)}{p^{2}\left(z_{0}\right)}-1\right\}
$$




$$
\begin{aligned}
& =(i a)^{\beta}\left(i \beta k \frac{1}{(i a)^{\beta}}-1\right) \\
& =a^{\beta} e^{i \pi \beta / 2}\left(e^{i \pi(1-\beta) / 2} \beta k \frac{1}{a^{\beta}}-1\right) .
\end{aligned}
$$

Hence we may write

$$
\begin{aligned}
\arg \left\{\frac{z_{0} p^{\prime}\left(z_{0}\right)}{p\left(z_{0}\right)}-p\left(z_{0}\right)\right\} & =\arg \left\{p\left(z_{0}\right)\left\{\frac{z_{0} p^{\prime}\left(z_{0}\right)}{p^{2}\left(z_{0}\right)}-1\right\}\right\} \\
& =\arg \left\{p\left(z_{0}\right\}\right)+\arg \left\{\frac{z_{0} p^{\prime}\left(z_{0}\right)}{p^{2}\left(z_{0}\right)}-1\right\} \\
& =\frac{\pi \beta}{2}+\arg \left\{e^{i \pi(1-\beta) / 2} \frac{\beta k}{a^{\beta}}-1\right\} \\
& \leq \frac{\pi \beta}{2}+\arg \left\{e^{i \pi(1-\beta) / 2}\left(\left(\frac{1+\beta}{1-\beta}\right)^{(1-\beta) / 2}+\left(\frac{1+\beta}{1-\beta}\right)^{-(1+\beta) / 2}\right)-1\right\} \\
& =\frac{\pi \beta}{2}+\tan ^{-1} \frac{\frac{\beta}{1-\beta}\left(\frac{1-\beta}{1+\beta}\right)^{(1+\beta) / 2} \sin \frac{\pi(1-\beta)}{2}}{-1+\frac{\beta}{1-\beta}\left(\frac{1-\beta}{1+\beta}\right)^{(1+\beta) / 2} \cos \frac{\pi(1-\beta)}{2}} \\
& =\frac{\pi \beta}{2}+\tan ^{-1} \frac{\beta n(\beta) \sin \frac{\pi(1-\beta)}{2}}{m(\beta)+\beta n(\beta) \cos \frac{\pi(1-\beta)}{2}},
\end{aligned}
$$

where

$$
m(\beta)=(1+\beta)^{(1+\beta) / 2}, \quad n(\beta)=(1-\beta)^{(\beta-1) / 2} .
$$

This contradicts the hypothesis (2.2) and for the case $p\left(z_{0}\right)=-\pi \beta / 2$, applying the same method as the above and Lemma 2.1, we can have

$$
\arg \left\{\frac{z_{0} p^{\prime}\left(z_{0}\right)}{p\left(z_{0}\right)}-p\left(z_{0}\right)\right\} \geq-\frac{\pi \beta}{2}-\tan ^{-1} \frac{\beta n(\beta) \sin \frac{\pi(1-\beta)}{2}}{m(\beta)+\beta n(\beta) \cos \frac{\pi(1-\beta)}{2}} .
$$

This contradicts the hypothesis (2.2) and therefore, it completes the proof of Theorem 2.1.

For $\beta=1 / 2$ Theorem 2.1 becomes the following corollary.

Corollary 2.2. Let $p(z)$ be in $\mathcal{H}[1,1]$, and suppose that

$$
\left|\arg \left\{\frac{z p^{\prime}(z)}{p(z)}-p(z)\right\}\right|>\frac{\pi}{2} \frac{2 \tan ^{-1}(1+\sqrt[4]{108})}{\pi}=\frac{\pi}{2}(0.85 \ldots)=1.3383 \ldots, \quad z \in \mathbb{D}
$$

then we have

$$
|\arg \{p(z)\}|<\frac{\pi}{4}, \quad z \in \mathbb{D} .
$$

For $p(z)=z f^{\prime}(z) / f(z), f(z) \in \mathcal{A}$, Theorem 2.1 becomes the following corollary. 
Corollary 2.3. Let $\beta$ be in $(0,1)$. Assume that $f(z) \in \mathcal{A}$ and $z f^{\prime}(z) / f(z) \in \mathcal{H}$ in $\mathbb{D}$, and that

$$
\left|\arg \left\{1+\frac{z f^{\prime \prime}(z)}{f^{\prime}(z)}-\frac{2 z f^{\prime}(z)}{f(z)}\right\}\right|>\frac{\pi \alpha(\beta)}{2}, \quad z \in \mathbb{D},
$$

then we have

$$
\left|\arg \left\{\frac{z f^{\prime}(z)}{f(z)}\right\}\right|<\frac{\pi \beta}{2}, \quad z \in \mathbb{D},
$$

where $\alpha(\beta)$ is defined in (2.4). This means that $f(z) \in \mathcal{S S}^{*}(\beta)$ or $f(z)$ is a strongly starlike function of order $\beta$, see (1.2).

Corollary 2.2 and 2.3 give together the following result.

Corollary 2.4. Assume that $f(z) \in \mathcal{A}$ and $z f^{\prime}(z) / f(z) \in \mathcal{H}$ in $\mathbb{D}$, and that

$$
\left|\arg \left\{1+\frac{z f^{\prime \prime}(z)}{f^{\prime}(z)}-\frac{2 z f^{\prime}(z)}{f(z)}\right\}\right|>\frac{\pi}{2} \frac{2 \tan ^{-1}(1+\sqrt[4]{108})}{\pi}=\frac{\pi}{2}(0.85 \ldots)=1.3383 \ldots,
$$

$z \in \mathbb{D}$, then we have

$$
\left|\arg \left\{\frac{z f^{\prime}(z)}{f(z)}\right\}\right|<\frac{\pi}{4}, \quad z \in \mathbb{D}
$$

or $f(z)$ is a strongly starlike function of order $1 / 2$, see (1.2).

Corollary 2.5. Assume that $f(z) \in \mathcal{A}$ and $z f^{\prime}(z) / f(z) \in \mathcal{H}$ in $\mathbb{D}$, and that

$$
\left|\arg \left\{\frac{z\left(2 f^{\prime \prime}(z)+z f^{\prime \prime \prime}(z)\right)}{2\left(f^{\prime}(z)+z f^{\prime \prime}(z)\right)}-\frac{z f^{\prime}(z)}{2 f(z)}-\sqrt{\frac{z f^{\prime}(z)}{f(z)}\left(1+\frac{z f^{\prime \prime}(z)}{f^{\prime}(z)}\right)}\right\}\right|>\frac{\pi \alpha(\beta)}{2}, \quad z \in \mathbb{D},
$$

then we have

$$
\left|\arg \left\{\sqrt{\frac{z f^{\prime}(z)}{f(z)}\left(1+\frac{z f^{\prime \prime}(z)}{f^{\prime}(z)}\right)}\right\}\right|<\frac{\pi \beta}{2}, \quad z \in \mathbb{D},
$$

where $\alpha(\beta)$ is defined in (2.4), or $f(z)$ is a $1 / 2$-strongly starlike functions of order $\beta$, see (1.6).

Theorem 2.6. [12] If $f(z)$ is analytic, $h(z)$ is convex univalent in $\mathbb{D}$, and that

$$
\left|\arg \left\{\frac{f^{\prime}(z)}{h^{\prime}(z)}\right\}\right| \leq \frac{\alpha \pi}{2}, \quad z \in \mathbb{D}
$$

for some $\alpha, 0 \leq \alpha \leq 1$, then

$$
\left|\arg \left\{\frac{f\left(z_{2}\right)-f\left(z_{1}\right)}{h\left(z_{2}\right)-h\left(z_{1}\right)}\right\}\right| \leq \frac{\alpha \pi}{2}
$$

for all $z_{1} \in \mathbb{D}$, and $z_{2} \in \mathbb{D}$. 
Notice that (2.10) implies that $f(z)$ satisfies Ozaki's univalence condition [11], or that $f(z)$ strongly close-to-convex of order $\alpha$ with respect to $g$. Recall [13], that $f(z) \in \mathcal{A}$ is said to be in the class $\mathcal{C}(\alpha)$ of strongly close-to-convex functions of order $\alpha, 0<\alpha \leq 1$, if and only if there exist $g \in \mathcal{K}, \varphi \in \mathbb{R}$, such that

$$
\left|\arg \left\{\frac{f^{\prime}(z)}{e^{i \varphi} g^{\prime}(z)}\right\}\right|<\frac{\pi \alpha}{2}, \quad z \in \mathbb{U} .
$$

If $\alpha=1$, then $\mathcal{C}(\alpha)$ becomes the well known class of close-to-convex functions, Kaplan [3]. Functions defined by (2.12) with $\varphi=0, \alpha=1$ where considered earlier by Ozaki [11], see also Umezawa [16, 17]. Moreover, Lewandowski $[4,5]$ defined the class of functions $f \in \mathcal{A}$ for which the complement of $f(\mathbb{U})$ with respect to the complex plane is a linearly accessible domain in the large sense. The Lewandowski's class is identical with the Kaplan's class $\mathcal{C}(1)$. It is worthy to note that $f \in \mathcal{A}$ satisfies the condition (2.11) with some convex univalent $g$ and $\alpha=1$ if and only if $f$ is close-to-convex function, see [2, p. 31].

Theorem 2.6 was improved to the following.

Theorem 2.7. [10] If $f(z)$ is analytic, $g(z)$ is convex univalent in $|z|<1, f^{\prime}(0)=g^{\prime}(0)$ and

$$
\left|\arg \left\{\frac{f^{\prime}(z)}{g^{\prime}(z)}\right\}\right| \leq \frac{\alpha \pi}{2}, \text { in }|z|<1,
$$

for some $\alpha, 0 \leq \alpha \leq 1$, then

$$
\left|\arg \left\{\frac{f\left(z_{2}\right)-f\left(z_{1}\right)}{g\left(z_{2}\right)-g\left(z_{1}\right)}\right\}\right|<\alpha\left(\frac{\pi}{2}-\log 2\right),\left|z_{1}\right|<1,\left|z_{2}\right|<1,
$$

for all $z_{1} \in \mathbb{D}$, and $z_{2} \in \mathbb{D}$, where $\pi / 2-\log 2=0.877649147 \ldots$

In this paper, we will improve Theorem 2.6 by adding some conditions.

Theorem 2.8. Let $f(z)=z+\sum_{n=2}^{\infty} a_{n} z^{n}$ be analytic in $\mathbb{D}, g(z)=z+\sum_{n=2}^{\infty} b_{n} z^{n}$ be analytic in $\mathbb{D}$, and suppose that

$$
\frac{g(z)}{z g^{\prime}(z)} \prec \frac{1+\delta z}{1-\delta z}, \quad z \in \mathbb{D}
$$

for some $\delta, 0<\delta \leq 1$, and

$$
\left|\arg \left\{\frac{f^{\prime}(z)}{g^{\prime}(z)}\right\}\right|<\frac{\pi}{2}\left\{\alpha+\frac{2}{\pi} \tan ^{-1} \frac{\alpha(1-\delta)^{2}}{1+2 \alpha \delta-\delta^{2}}\right\}, \quad z \in \mathbb{D},
$$

and

$$
\tan \frac{\alpha \pi}{2} \leq \frac{(1-\delta)^{2}}{2 \delta}
$$

for some $\alpha, 0<\alpha<1$. Then we have

$$
\left|\arg \left\{\frac{f(z)}{g(z)}\right\}\right| \leq \frac{\alpha \pi}{2}, \quad z \in \mathbb{D}
$$


Proof. Let us put

$$
p(z)=\frac{f(z)}{g(z)}, p(0)=1, \quad z \in \mathbb{D} .
$$

Then it follows that

$$
f(z)=p(z) g(z), \quad f^{\prime}(z)=g^{\prime}(z) p(z)+g(z) p^{\prime}(z)
$$

and

$$
\frac{f^{\prime}(z)}{g^{\prime}(z)}=p(z)+\frac{g(z)}{g^{\prime}(z)} p^{\prime}(z)=p(z)\left(1+\frac{g(z)}{z g^{\prime}(z)} \frac{z p^{\prime}(z)}{p(z)}\right) .
$$

From the hypothesis, we have

$$
\frac{1-\delta}{1+\delta}<\mathfrak{R e}\left\{\frac{g(z)}{z g^{\prime}(z)}\right\}<\frac{1+\delta}{1-\delta}, \quad z \in \mathbb{D}
$$

and

$$
\frac{-2 \delta}{1-\delta^{2}}<\mathfrak{I m}\left\{\frac{g(z)}{z g^{\prime}(z)}\right\}<\frac{2 \delta}{1-\delta^{2}}, \quad z \in \mathbb{D} .
$$

If there exists a point $z_{0}$ with $\left|z_{0}\right|<1$, such that $|\arg \{p(z)\}|<\pi \alpha / 2$ for $|z|<\left|z_{0}\right|$ and $\left|\arg \left\{p\left(z_{0}\right)\right\}\right|=\pi \alpha / 2$ for some $0<\alpha<1$, then by Lemma 2.1 we have

$$
\frac{z_{0} p^{\prime}\left(z_{0}\right)}{p\left(z_{0}\right)}=\frac{2 i k \arg \left\{p\left(z_{0}\right)\right\}}{\pi}
$$

for some

$$
k \geq\left(a+a^{-1}\right) / 2
$$

where

$$
\left\{p\left(z_{0}\right)\right\}^{1 / \alpha}= \pm i a
$$

and $a>0$. Then, for the case $p\left(z_{0}\right)=a^{\alpha}(\cos (\pi \alpha / 2)+i \sin (\pi \alpha / 2))$, we have from (2.21)

$$
\arg \left\{\frac{f^{\prime}\left(z_{0}\right)}{g^{\prime}\left(z_{0}\right)}\right\}=\arg \left\{p\left(z_{0}\right)\left(1+\frac{g\left(z_{0}\right)}{z_{0} g^{\prime}\left(z_{0}\right)} \frac{z_{0} p^{\prime}\left(z_{0}\right)}{p\left(z_{0}\right)}\right)\right\}
$$

Notice that if we assume $\arg \{w\} \in[0, \pi]$, then under the hypothesis of Theorem 2.8, we may apply the formula

$$
\arg \left\{p\left(z_{0}\right)\left(1+\frac{g\left(z_{0}\right)}{z_{0} g^{\prime}\left(z_{0}\right)} \frac{z_{0} p^{\prime}\left(z_{0}\right)}{p\left(z_{0}\right)}\right)\right\}=\arg \left\{p\left(z_{0}\right)\right\}+\arg \left\{1+\frac{g\left(z_{0}\right)}{z_{0} g^{\prime}\left(z_{0}\right)} \frac{z_{0} p^{\prime}\left(z_{0}\right)}{p\left(z_{0}\right)}\right\} .
$$

It is because of that

$$
\begin{aligned}
& \arg \left\{p\left(z_{0}\right)\right\} \in[0, \pi], \\
& \arg \left\{1+\frac{g\left(z_{0}\right)}{z_{0} g^{\prime}\left(z_{0}\right)} \frac{z_{0} p^{\prime}\left(z_{0}\right)}{p\left(z_{0}\right)}\right\} \in[0, \pi],
\end{aligned}
$$




$$
\arg \left\{p\left(z_{0}\right)\right\}+\arg \left\{1+\frac{g\left(z_{0}\right)}{z_{0} g^{\prime}\left(z_{0}\right)} \frac{z_{0} p^{\prime}\left(z_{0}\right)}{p\left(z_{0}\right)}\right\} \in[0, \pi],
$$

where (2.24) holds because of (2.17), namely

$$
\begin{aligned}
& \arg \left\{p\left(z_{0}\right)\right\}+\arg \left\{1+\frac{g\left(z_{0}\right)}{z_{0} g^{\prime}\left(z_{0}\right)} \frac{z_{0} p^{\prime}\left(z_{0}\right)}{p\left(z_{0}\right)}\right\} \in[0, \pi] \\
\Leftrightarrow & \frac{\alpha \pi}{2}+\pi-\tan ^{-1} \frac{\alpha \frac{1-\delta}{1+\delta}}{\alpha \frac{2 \delta}{1-\delta^{2}}} \in[0, \pi] \\
\Leftrightarrow & \frac{\alpha \pi}{2}+\pi-\tan ^{-1} \frac{\frac{1-\delta}{1+\delta}}{\frac{2 \delta}{1-\delta^{2}}} \in[0, \pi) \\
\Leftrightarrow & 0 \leq \frac{\alpha \pi}{2}+\pi-\tan ^{-1} \frac{\frac{1-\delta}{1+\delta}}{\frac{2 \delta}{1-\delta^{2}}} \leq \pi \\
\Leftrightarrow & 0 \leq \tan ^{-1} \frac{\frac{1-\delta}{1+\delta}}{\frac{2 \delta}{1-\delta^{2}}}-\frac{\alpha \pi}{2} \leq \pi \\
\Leftrightarrow & \frac{\alpha \pi}{2} \leq \tan ^{-1} \frac{\frac{1-\delta}{1+\delta}}{\frac{2 \delta}{1-\delta^{2}}} \\
\Leftrightarrow & \frac{\alpha \pi}{2} \leq \tan ^{-1} \frac{(1-\delta)^{2}}{2 \delta} \\
\Leftrightarrow & \tan \frac{\alpha \pi}{2} \leq \frac{(1-\delta)^{2}}{2 \delta} .
\end{aligned}
$$

Therefore, we have

$$
\begin{aligned}
\arg \left\{\frac{f^{\prime}\left(z_{0}\right)}{g^{\prime}\left(z_{0}\right)}\right\} & =\arg \left\{p\left(z_{0}\right)\right\}+\arg \left\{1+\frac{g\left(z_{0}\right)}{z_{0} g^{\prime}\left(z_{0}\right)} \frac{z_{0} p^{\prime}\left(z_{0}\right)}{p\left(z_{0}\right)}\right\} \\
& =\frac{\alpha \pi}{2}+\arg \left\{1+i \alpha k \frac{g\left(z_{0}\right)}{z_{0} g^{\prime}\left(z_{0}\right)}\right\} .
\end{aligned}
$$

We know that

$$
\frac{g\left(z_{0}\right)}{z_{0} g^{\prime}\left(z_{0}\right)}
$$

lies in a rectangle described by (2.21), (2.22) in the right half-plane. Therefore,

$$
\left\{i \alpha \frac{g\left(z_{0}\right)}{z_{0} g^{\prime}\left(z_{0}\right)}\right\}
$$

lies in a rectangle in the upper half-plane and

$$
-\alpha \frac{2 \delta}{1-\delta^{2}}<\mathfrak{R e}\left\{i \alpha \frac{g(z)}{z g^{\prime}(z)}\right\}<-\alpha \frac{-2 \delta}{1-\delta^{2}}, \quad z \in \mathbb{D}
$$

and

$$
\alpha \frac{1-\delta}{1+\delta}<\mathfrak{I m}\left\{i \alpha \frac{g(z)}{z g^{\prime}(z)}\right\}<\alpha \frac{1+\delta}{1-\delta}, \quad z \in \mathbb{D} .
$$


This gives

$$
1-\alpha \frac{2 \delta}{1-\delta^{2}}<\mathfrak{R e}\left\{1+i \alpha \frac{g(z)}{z g^{\prime}(z)}\right\}<1+\alpha \frac{2 \delta}{1-\delta^{2}}, \quad z \in \mathbb{D}
$$

and

$$
\alpha \frac{1-\delta}{1+\delta}<\mathfrak{I m}\left\{1+i \alpha \frac{g(z)}{z g^{\prime}(z)}\right\}<\alpha \frac{1+\delta}{1-\delta}, \quad z \in \mathbb{D},
$$

and so

$$
\tan ^{-1} \frac{\alpha \frac{1-\delta}{1+\delta}}{1+\alpha \frac{2 \delta}{1-\delta^{2}}} \leq \arg \left\{1+i \alpha k \frac{g\left(z_{0}\right)}{z_{0} g^{\prime}\left(z_{0}\right)}\right\} .
$$

Inequality (2.28) together with (2.25) contradicts condition (2.16). For the case $\arg \left\{p\left(z_{0}\right)\right\}=$ $-\pi \alpha / 2$, applying the same method as above we can get

$$
\arg \left\{\frac{f\left(z_{0}\right)}{g\left(z_{0}\right)}\right\} \leq \frac{\pi}{2}\left\{\alpha+\frac{2}{\pi} \tan ^{-1} \frac{\alpha(1-\delta)^{2}}{1+2 \alpha \delta-\delta^{2}}\right\} .
$$

This is also contradicts hypothesis and therefore it completes the proof of Theorem 2.8.

Observe that condition (2.15) satisfies the function

$$
g(z)=\frac{z}{(1+\delta z)^{2}}
$$

so Theorem 2.8 becomes the following corollary.

Corollary 2.9. Let $f(z)=z+\sum_{n=2}^{\infty} a_{n} z^{n}$ be analytic in $\mathbb{D}, g(z)=z+\sum_{n=2}^{\infty} b_{n} z^{n}$ be analytic in $\mathbb{D}$, and suppose that

$$
\left|\arg \left\{(1-\delta z) \frac{f^{\prime}(z)}{(1+\delta z)^{3}}\right\}\right|<\frac{\pi}{2}\left\{\alpha+\frac{2}{\pi} \tan ^{-1} \frac{\alpha(1-\delta)^{2}}{1+2 \alpha \delta-\delta^{2}}\right\}, \quad z \in \mathbb{D},
$$

and

$$
\tan \frac{\alpha \pi}{2} \leq \frac{(1-\delta)^{2}}{2 \delta}
$$

for some $\alpha, 0<\alpha<1$. Then we have

$$
\left|\arg \left\{(1+\delta z)^{2} \frac{f(z)}{z}\right\}\right| \leq \frac{\alpha \pi}{2}, \quad z \in \mathbb{D} .
$$

For $\alpha=1 / 2$ and $\delta=2-\sqrt{3}$ Theorem 2.8 becomes the following corollary.

Corollary 2.10. Let $f(z)=z+\sum_{n=2}^{\infty} a_{n} z^{n}$ be analytic in $\mathbb{D}, g(z)=z+\sum_{n=2}^{\infty} b_{n} z^{n}$ be analytic in $\mathbb{D}$, and suppose that

$$
\frac{g(z)}{z g^{\prime}(z)} \prec \frac{1+(2-\sqrt{3}) z}{1-(2-\sqrt{3}) z}, \quad z \in \mathbb{D}
$$


and

$$
\left|\arg \left\{\frac{f^{\prime}(z)}{g^{\prime}(z)}\right\}\right|<\frac{\pi}{2}\left\{\frac{1}{2}+\frac{2}{\pi} \tan ^{-1} \frac{2 \sqrt{3}-1}{11}\right\}=\frac{\pi}{2} \cdot 0.64 \ldots, \quad z \in \mathbb{D},
$$

then we have

$$
\left|\arg \left\{\frac{f(z)}{g(z)}\right\}\right| \leq \frac{\pi}{4}, \quad z \in \mathbb{D} .
$$

\section{References}

[1] D. A. Brannan and W. E. Kirwan, On some classes of bounded univalent functions, J. London Math. Soc. 1(1969)(2) 431-443.

[2] A. W. Goodman, Univalent Functions, Vol. II, Mariner Publishing Co.: Tampa, Florida (1983).

[3] W. Kaplan, Close to convex schlicht functions, Michigan Math. J. 1(1952) 169-185.

[4] Z. Lewandowski, Sur l'identité de certaines classes de fonctions univalentes, I. Ann. Univ. Mariae Curie-Skłodowska Sect. A 12(1958) 131-146.

[5] Z. Lewandowski, Sur l'identité de certaines classes de fonctions univalentes, II. Ann. Univ. Mariae Curie-Skłodowska Sect. A 14(1960) 19-46.

[6] Z. Lewandowski, S. Miller, E. Złotkiewicz, Gamma starlike functions, Ann. Univ. Mariae Curie-Skłodowska, Sect. A 28(1974) 53-58.

[7] M. Nunokawa, On Properties of Non-Carathéodory Functions, Proc. Japan Acad. Ser. A 68(6)(1992) 152-153.

[8] M. Nunokawa, On the order of strongly starlikeness of strongly convex functions, Proc. Japan Acad. Ser. A 69(7)(1993) 234-237.

[9] M. Nunokawa and J. Sokól, Strongly gamma-starlike functions of order alpha, Ann. Univ. Mariae Curie-Skłodowska, Sect. A 67(2)(2013) 43-51.

[10] M. Nunokawa and J. Sokół, On an extension of Sakaguchi's result, Journal of Mathematical Inequalities, 9(3)(2015) 683-697.

[11] S. Ozaki, On the theory of multivalent functions, Sci. Rep. Tokyo Bunrika Daig. A2(1935) $167-188$.

[12] Ch. Pommerenke, On close-to-convex analytic functions, Trans. Amer. Math. Soc. 114(1)(1965) 176-186. 
[13] M. Reade, The coefficients of close-to-convex functions, Duke Math. J. 23(1956) 459-462.

[14] M. S. Robertson, On the theory of univalent functions, Ann. Math. 37(1936) 374-408.

[15] J. Stankiewicz, Quelques problèmes extrèmaux dans les classes des fonctions $\alpha$ angulairement ètoilèes, Ann. Univ. Mariae Curie-Skłodowska, Sect. A 20(1966) 59-75.

[16] T. Umezawa, On the theory of univalent functions, Tohoku Math. J. 7(1955) 212-228.

[17] T. Umezawa, Multivalently close-to-convex functions, Proc. Amer. Math. Soc. 8(1957) 869874.

[18] D. R. Wilken and J. Feng, A remark on convex and starlike functions, J. London Math. Soc. 21(1980)(2) 287-290.

Mamoru Nunokawa University of Gunma, Hoshikuki-cho 798-8, Chuou-Ward, Chiba, 2600808, Japan

E-mail: mamoru_nuno@doctor.nifty.jp

Janusz Sokół University of Rzeszów, College of Natural Sciences, ul. Prof. Pigonia 1, 35-310 Rzeszów, Poland

E-mail: jsokol@ur.edu.pl 